\title{
Socioeconomic Status, Social Support, Age, and Health
}

\author{
TONI C. ANTONUCCI, ${ }^{a}$ KRISTINE J. AJROUCH, AND MARY JANEVIC \\ Institute for Social Research, The University of Michigan, \\ 426 Thompson Street, P.O. Box 1248, Ann Arbor, Michigan 48106, USA
}

This study examines the hypothesis that aspects of social relations moderate the relationship between SES and health; that is, this association varies with differences along four major dimensions of social relations: instrumental, emotional, and negative support, and network structure.

In 1992, 923 white and black respondents aged 40 and over were interviewed as part of the Survey of Social Relations. ${ }^{1}$ The sample was drawn from a stratified probability sample in the Detroit metropolitan area. Social relations variables included the number of people in social network, perceived instrumental support (whether spouse and child would provide care if respondent was ill), emotional support (whether respondent confides in spouse and child), and negative support (whether spouse and child get on the respondent's nerves). Respondents rated all social support variables on a five-point scale (1, strongly agree; 5 , strongly disagree). Education level was used as an SES indicator. Years of education were split into three groups: less than high school, high school, and more than high school. Health was measured by a global self-rated health question (5, poor; 1 , excellent).

Following Baron and Kenny, ${ }^{2}$ we assessed the moderating impact of the social relations variables on the relationship between SES and health by regressing health on (1) the independent SES variable (education level), (2) the social relations variable that is hypothesized to be the moderator, and (3) the interaction of (1) and (2). A significant interaction term implies that the social relations variable is moderating the impact of SES on health. Analyses were conducted separately for each of two age groups: 40-59 and 60-93.

Findings indicate that there is no interaction effect of SES and social network size on health. However, interaction effects are evident for instrumental, emotional, and negative support. These are summarized in TABLE 1. Graphs of the significant interactions suggest complex associations among SES, social relations variables, and health. For example, FIGURE 1 demonstrates that respondents aged 40-59 with more than a high school education and who confide in their spouse are healthier than those who do not. This relationship was reversed among respondents with less than a high school education. However, in the 60-93 age group, health is about the same for all respondents who confide in their spouse. Among those in this age group who do not confide in their spouse, education level has a negative effect on health: people with less than a high school education report poorer health; those with more than a high school education report better health. A consistent pattern that emerged was that respondents in the middle education group (high school) were the least likely in both

${ }^{a}$ Address for correspondence: $313-747-4575$ (voice). 
TABLE 1. Interactions between social relations and SES

\begin{tabular}{lcc}
\hline Social relations & \multicolumn{2}{c}{ Age } \\
\cline { 2 - 3 } & $40-59$ & $60-93$ \\
\hline Network structure & $\mathrm{ns}$ & $\mathrm{ns}$ \\
Network size & & \\
Instrumental & $p<0.001$ & $\mathrm{~ns}$ \\
Spouse & $p<0.01$ & $\mathrm{~ns}$ \\
Child & & \\
Emotional & $p<0.001$ & $\mathrm{n}<0.05$ \\
Spouse & $p<0.05$ & $\mathrm{~ns}$ \\
Child & & $p<0.05$ \\
Negative & $\mathrm{ns}$ & $\mathrm{ns}$ \\
Spouse & $p<0.05$ & \\
Child & & \\
\hline
\end{tabular}

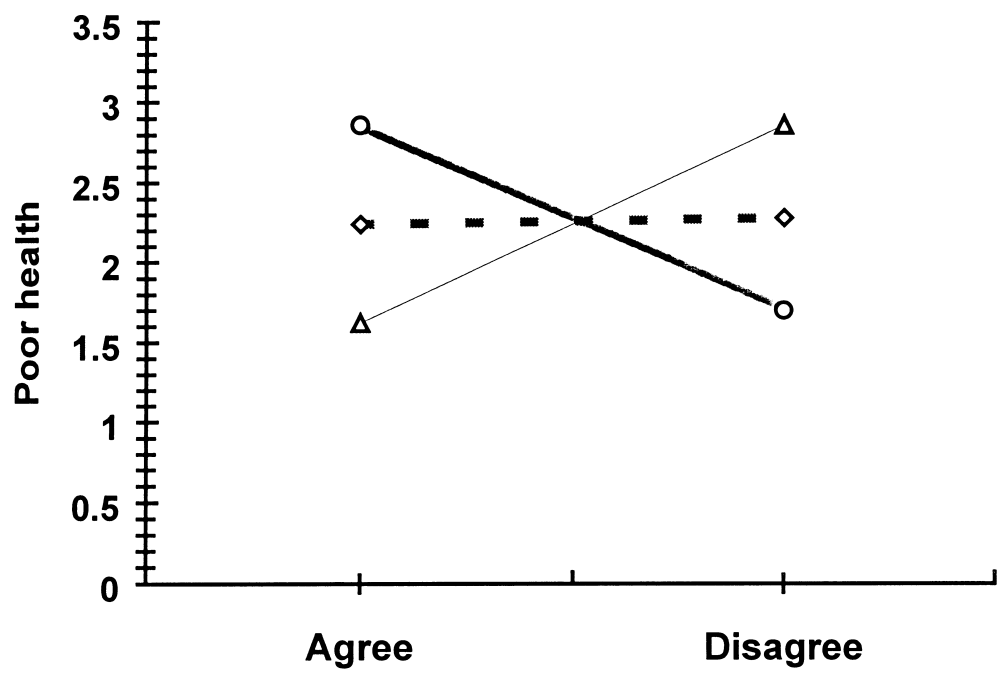

FIGURE 1. Emotional support, ages 40-59: confide in spouse $(p<0.001)$. Symbols: $\bigcirc$, below high school; $\diamond$, high school; $\triangle$, above high school. 
age groups and across variables to experience a moderating effect of social relations on health.

The results suggest that social relations affect the association between SES and health across age. Of special interest are those in the lowest SES group. Older, lower SES adults who do not confide in their spouse are clearly more vulnerable to health problems, but younger people who confide in their spouse are less healthy. This latter finding might speak to the special vulnerability of younger individuals who face significant health problems. Social relations are apparently more likely to exert a moderating effect on the SES-health relationship in the 40-59 age group than in the 6093 age group, a possible life course effect. Additionally, the effect of SES on health for those considered middle aged (40-59) is moderated by both the relationship with their children and spouse, whereas only relations with the spouse had a significant moderating effect for the older (60-93) respondents.

In sum, these findings suggest that it is necessary to recognize that types of social relations differentially affect health among people of different ages and education levels. Future research should examine other SES indicators as well as other types of social relations across the life span.

\section{REFERENCES}

1. Antonucci, T.C. \& H. Akiyama. 1996. Social Relations and Mental Health Over the Life Course, Final Report. National Institutes of Mental Health, \#ROIMH46549.

2. BARON, R.M. \& D.A. KENNY. 1986. Moderator-mediator variable distinction in social psychological research: conceptual, strategic and statistical considerations. J. Pers. Soc. Psychol. 51: 1173-1182. 